\title{
Arabidopsis transcriptome changes prior and post environmental stresses
}

\section{Editorial}

Because plants experience diverse environmental stresses which negatively affect development and productivity during their lifetime, they deploy certain strategies to adapt to these threats. One of these strategies is the modulation of gene expression levels during stress conditions. To study the plant response to biotic and abiotic stresses, individually or in combinations, numerous transcriptomics studies analyzing variations in gene expression have been performed. There are different technologies used for transcriptomics studies including hybridization-based and sequence based approaches. ${ }^{1,2}$ The hybridization-based approach, also known as microarray technology, is used for gene expression profiling. ${ }^{3}$ This technology is dependent on pre-existing knowledge about genome sequences; which is opposite to the sequence-based approach (i.e. RNA-seq or ChIP-seq) which does not rely on a reference genome to determine the cDNA sequences directly. Recent advances in high throughput transcriptomics have led to a significant increase in generating data with more emphasis on transcriptomics variation in response to an individual treatment than on combinations of treatments.

In attempts to analyze gene expression changes in plants infected with pathogens and treated with abiotic stresses, the Arabidopsis transcriptome response to dehydration and the plant parasitic nematode, Heterodera schachtii, was performed using microarray. ${ }^{4}$ In addition, analysis of the transcript profiles on Arabidopsis plants treated with flagellin, cold, heat, high light and salt did not only detect single stress responses, but also the shared responses between combinations of biotic and abiotic stresses. ${ }^{5}$ Recently, two reports on the transcriptome and co-expression network analyses after infection with Botrytis cinerea and treatments with cold, heat, drought, salinity, osmotic and oxidative stresses individually and in combination, identified potential regulatory genes in response to environmental stress. ${ }^{6,7}$ In the latter studies, the combinations of the expression of $B$. cinerea-upregulated genes $(B U G s)$ and $B$. cinerea-downregulated genes $(B D G s)$ with each of the six classes of environmental stresses were analyzed. It was found that $2.5 \%, 6 \%, 12 \%, 19 \%, 25 \%$ or $41 \%$ of the $B U G s$ were also induced by heat, drought, oxidative stress, salinity, cold or osmotic stress, respectively. On the other hand, the percentage of co-downregulated genes between $B$. cinerea and the same abiotic stresses were $7.6 \%$ heat, $6.8 \%$ drought, $5.5 \%$, oxidative stress, $18.9 \%$ salinity, $33 \%$ cold and $47.8 \%$ osmotic stress. Overall, there were only $0.2 \%$ of the $B U G s$ showed common induction and $1.1 \%$ of the $B D G$ s showed common repressions with all six abiotic stress treatments.

Efforts using transcriptomics have also shed light on the function of regulated $\square$ genes encoding proteins that have various roles in plant defense. Botrytis $\square$ induced Kinase 1 (BIK1), WRKY33, WRKY70, and Expansin $\square$ like $A 2$ (EXLA2) are examples of regulated $\square$ genes in response to $B$. cinerea that have been previously identified by microarray. ${ }^{8-11}$ RNA $\square$ seq analysis determines the global impact and specific targets of MED18 modulating the plant immunity, flowering time and responses to hormones through interactions with distinct transcription factors. ${ }^{12}$
Volume 2 Issue 4 - 2015

\author{
Synan AbuQamar \\ Department of Biology, United Arab Emirates University, United \\ Arab Emirates \\ Correspondence: Synan AbuQamar, Department of Biology, \\ United Arab Emirates University, Al-Ain, United Arab Emirates, \\ Tel +97I (3) 7|3-6733, Fax +97l (3) 7|3-4927, \\ Email sabuqamar@uaeu.ac.ae
}

Received: June 03, 20I5 | Published: June 04, 2015

The combination of biotic and abiotic stresses activates the expression of unique and common sets of genes that is orchestrated by hormonal or non-hormonal pathways. Recently, the droughtinduced gene, Responsive to Dehydration (RD20), is induced by the necrotrophic fungi, Alternaria brassicicola and B. cinerea, and abiotic stresses. ${ }^{6,7,13}$ The $r d 20$ mutant plants exhibited increased sensitivity to necrotrophic fungi and drought. AbuQamar et al., ${ }^{8}$ have shown that mutations in Arabidopsis expansin-like A2 (EXLA2) increased resistance to necrotrophic fungi, but hypersensitivity to salt and cold. It was also shown that cyclopentenone accumulated after $B$. cinerea attack leading to EXLA2 repression. Both BIK1 and WRKY33 play an antagonistic role in plant defense contributing as positive and negative regulators to resistance to $B$. cinerea and the bacterial pathogen Pseudomonas syringae pv tomato, respectively, ${ }^{9,10}$ suggesting a crosstalk between jasmonic acid- and salicylic acidregulated disease response pathways. The identification of Arabidopsis Botrytis Susceptible 1 interactors (BOI) and BIK1 regulators uncovers the function of these interactors and regulators in plant responses to pathogen infection and abiotic stress. ${ }^{14,15}$ Overall, the purpose of such transcriptomics research is to promote an adaptation to shortand long $\square$ term environmental changes, to identify a map of shared stress $\square$ regulated genes, and ultimately to further our understanding toward plant responses to multiple stresses.

\section{Acknowledgements}

S. AbuQamar was supported with funds from the Khalifa Center for Biotechnology and Genetic Engineering $\square$ UAEU [KCGEB $\square 2 \square 2013$ ].

\section{Conflict of interest}

The author declares no conflict of interest.

\section{References}

1. Tan KC, Ipcho SVS, Trengove RD, et al. Assessing the impact of transcriptomics, proteomics and metabolomics on fungal phytopathology. Mol Plant Pathol. 2009;10(5):703-715.

2. Wang Z, Gerstein M, Snyder M. RNA-Seq:a revolutionary tool for transcriptomics. Nat Rev Genet. 2009;10(1):57-63. 
3. Nowrousian M. Of patterns and pathways:microarray technologies for the analysis of filamentous fungi. Fungal Biol Rev. 2007;21(4):171178.

4. Atkinson NJ, Lilley CJ, Urwin PE. Identification of genes involved in the response of Arabidopsis to simultaneous biotic and abiotic stresses. Plant Physiol. 2013;162(4):2028-2041.

5. Rasmussen S, Barah P, Suarez-Rodriguez MC, et al. Transcriptome responses to combinations of stresses in Arabidopsis. Plant Physiol. 2013;161(4):1783-1794.

6. Sham A, Al-Azzawi A, Al-Ameri S, et al. Transcriptome analysis reveals genes commonly induced by Botrytis cinereainfection, cold, drought and oxidative stresses in Arabidopsis. PLoS One. 2014;9(11):e113718.

7. Sham A, Moustafa K, Al-Ameri S, et al. Identification of Arabidopsis Candidate Genes in Response to Biotic and Abiotic Stresses Using Comparative Microarrays. PLOS ONE. 2015;10(5):e125666.

8. Abuqamar S, Ajeb S, Sham A, et al. A mutation in the expansinlike A2gene enhances resistance to necrotrophic fungi and hypersensitivity to abiotic stress in Arabidopsis thaliana. Mol Plant Pathol. 2013;14(8):813-827.

9. Veronese P, Nakagami H, Bluhm B, et al. The membrane-anchored BOTRYTIS INDUCED KINASE1 has distinct roles in Arabidopsis resistance to necrotrophic and biotrophic pathogens. Plant Cell. 2006;18(1):257-273.
10. Zheng Z, Qamar SA, Chen Z, et al. Arabidopsis WRKY33 transcription factor is required for resistance to necrotrophic fungal pathogens. Plant J. 2006;48(4):592-605.

11. AbuQamar S, Chen X, Dhawan R, et al. Expression profiling and mutant analysis reveals complex regulatory networks involved in Arabidopsis response to B. cinerea infection. Plant J. 2006;48(1):2844.

12. Lai A, Schluttenhofer CM, Bhide K, et al. MED18 interaction with distinct transcription factors regulates multiple plant functions. Nat Commun. 2014;5:3064

13. Hanano A, Bessoule J-J, Heitz T, et al. Involvement of the caleosin/ peroxygenase RD20 in the control of cell death during Arabidopsis responses to pathogens. Plant Signal Behav. 2015;10(3):e991574.

14. Luo H, Laluk K, Lai Z, et al. The Arabidopsis Botrytis Susceptible1 Interactor defines a subclass of RING E3 ligases that regulate pathogen and stress responses. Plant Physiol. 2010;154(4):17661782 .

15. Laluk $\mathrm{K}$, Luo $\mathrm{H}$, Chai $\mathrm{M}$, et al. Biochemical and genetic requirements for function of the immune response regulator BOTRYTIS-INDUCED KINASE1 in plant growth, ethylene signaling, and PAMP-triggered immunity in Arabidopsis. Plant Cell. 2011;23(8):2831-2849. 